\title{
False Positive Reduction in Mammographic Mass Detection Using Local Binary Patterns
}

\author{
Arnau Oliver, Xavier Lladó, Jordi Freixenet, and Joan Martí \\ Institute of Informatics and Applications - University of Girona \\ Campus Montilivi, Ed. P-IV, 17071, Girona, Spain \\ \{aoliver, llado, jordif, joanm\}@eia.udg.es
}

\begin{abstract}
In this paper we propose a new approach for false positive reduction in the field of mammographic mass detection. The goal is to distinguish between the true recognized masses and the ones which actually are normal parenchyma. Our proposal is based on Local Binary Patterns (LBP) for representing salient micro-patterns and preserving at the same time the spatial structure of the masses. Once the descriptors are extracted, Support Vector Machines (SVM) are used for classifying the detected masses. We test our proposal using a set of 1792 suspicious regions of interest extracted from the DDSM database. Exhaustive experiments illustrate that LBP features are effective and efficient for false positive reduction even at different mass sizes, a critical aspect in mass detection systems. Moreover, we compare our proposal with current methods showing that LBP obtains better performance.
\end{abstract}

\section{Introduction}

Breast cancer is one of the most devastating and deadly diseases for women in their 40s. It is estimated that between one in eight and one in twelve women will develop breast cancer during their lifetime [1. The most used method to detect breast cancer is mammography, because it allows the detection of the cancer at its early stages, a crucial issue for a high survival rate. The introduction of digital mammography gave the opportunity of increasing the number of commercial Computer-Aided Detection systems to help radiologists to interpret and diagnose mammograms. During the last decade several algorithms have been proposed for the automatic mass detection purpose [2]. However, the main drawback of these methods is the high number of obtained false positives [3]. A false positive is a region being normal tissue but interpreted by the automatic algorithm as a suspicious one. The so called false positive reduction algorithms try to solve this drawback, i.e. given a Region of Interest (RoI) - a sub-image containing the suspicious region - the aim is to validate whether it contains a real lesion or it is only a region depicting normal parenchyma.

Different algorithms have been proposed so far for such a task. For instance, the work of Sahiner et al. 4] consisted in extracting a huge set of features, selecting the most discriminative ones using genetic algorithms, and then classifying by using linear classifiers or neural networks. A similar strategy was used by Christoyianni et al. [5], who extracted gray-level, texture, and features related 


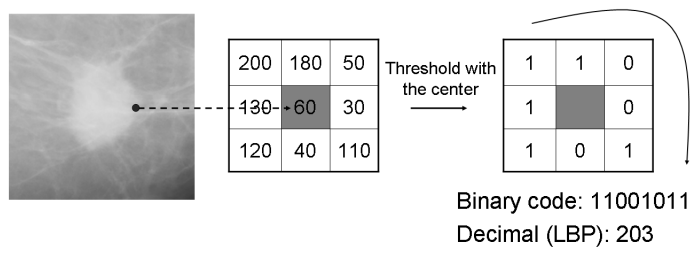

Fig. 1. Example of the basic LBP operator

to independent component analysis to train a neural network. Qian et al. 6] analyzed the implementation of an adaptive module to improve a Kalman-filter based neural net using features obtained from a wavelet decomposition. The works of Chang et al. 7] and Tourassi et al. 8] were based on directly comparing a new RoI image with all the RoI images in the database. In the first one, the gray level and the shape were used as a likelihood measure, while on the second one the similarity was based on mutual information. A different strategy was recently proposed by Oliver et al. 910, who adapted the eigenfaces approach to the mass detection problem.

In this paper we propose an alternative approach to perform mass false positive reduction by using the textural properties of the masses. The idea of our proposal is inspired by the recent work of Ahonen et al. [11 in which Local Binary Patterns (LBP) are successfully applied to the face recognition problem. In our work, we propose the use of LBP operators 12 to characterize micropatterns (i.e. edges, lines, spots, flat areas) and preserve at the same time the spatial structure of the mass. To our knowledge this is the first attempt to use LBP in the field of mammographic mass detection. Once the LBP characterization is done, we use Support Vector Machines to classify the RoIs between real masses and normal parenchyma. We perform experiments on a complete set of 1792 RoIs extracted from the DDSM database, evaluating the results when using different RoI image sizes, and when using different ratios of number of RoIs depicting masses and RoIs depicting normal tissue in the database. The obtained results and the comparison with previous works demonstrate the validity of our approach for reducing false positives.

The rest of the paper is organized as follows. Section 2 briefly presents the LBP as a texture descriptor. Section 3 describes our approach for mass false positive reduction. Experimental results are presented in Section 4 Finally, the paper ends with conclusions.

\section{Local Binary Patterns (LBP)}

The original LBP operator was introduced by Ojala et al. 13 with the idea to perform gray scale invariant two-dimensional texture analysis. The LPB operator labels the pixels of an image by thresholding the neighborhood (i.e. $3 \times 3$ ) of each pixel with the center value and considering the result of this thresholding as a binary number. Figure 1 shows an example of how to compute a LBP code. When all the pixels have been labeled with the corresponding LBP codes, the 
histogram of the labels is computed and used as a texture descriptor. Initially, the limitation of this basic LBP operator was its small $3 \times 3$ neighborhood since it can not deal with dominant features with large scale structures. Due to this fact, the operator was later extended to use neighborhoods of different sizes 12. The idea of this operator is the detection of local binary patterns at circular neighborhoods of any quantization of the angular space and at any spatial resolution. Therefore, it is possible to derive the operator for a general case based on a circularly symmetric neighborhood of $P$ members on a circle of radius $R$. In addition to evaluating the performance of individual operators of a particular configuration $(P, R)$, one could analyze and combine responses of multiple operators realized with different parameters $(P, R)$.

Another extension of LBP was the use of the so called uniform patterns [12. A LBP is called uniform if it contains at most two bitwise transitions from 0 to 1 or vice versa when the binary string is considered circular. For example, 000011100 and 11100011 are uniform patterns. As stated by Ojala et al., the uniform patterns account for nearly $90 \%$ of all patterns in the $(8,1)$ neighborhood and for about $70 \%$ in the $(16,2)$ neighborhood in texture images. In this paper, we shall refer the uniform LBP operator as $L B P_{P, R}^{u 2}$, where the subscript represents using the operator in a $(P, R)$ neighborhood and the superscript $u 2$ indicates using uniform patterns.

As in the original work of Ojala et al. the histogram of the labeled image $f_{l}(x, y)$ is used as a descriptor. We can define this histogram as

$$
H_{i}=\sum_{x, y} I\left(f_{l}(x, y)=i\right), \quad i=0, \ldots, n-1
$$

where $n$ is the number of different labels produced by the $L B P$ operator, and $I(A)=1$ when $A$ is true, while $I(A)=0$ when $A$ is false.

This discrete occurrence histogram of the uniform patterns computed over an image or an image region contains information about the distribution of the local micro-patterns such as edges, spots and flat areas, and has been demonstrated to be a very powerful texture descriptor.

\section{Using LBP for Mammographic False Positive Reduction}

In this work, we are dealing with mammographic images and in particular with the mass false positive reduction problem. Inherently to these images, texture and its spatial information play a key role in correctly detecting the masses. Due to this fact, we base our approach on the use of LBP for representing salient micro-patterns, and an adaptation of these descriptors for preserving also the spatial structure of the masses. The idea of our proposal has been inspired by the recent work of Ahonen et al. [1] in which LBP is used to perform face recognition. Our general procedure consists in using the LBP texture descriptor to build several local descriptions of the RoI and combining them into a global description. Afterwards, this global LBP descriptor is the one used to reduce the 


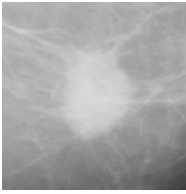

(a)

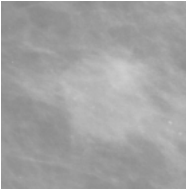

(b)

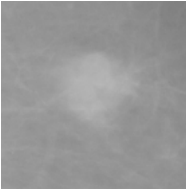

(c)

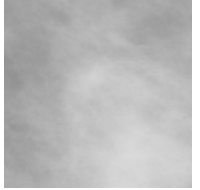

(d)

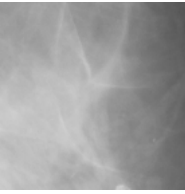

(e)

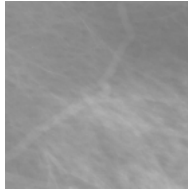

(f)

Fig. 2. Examples of RoIs: (a-c) RoIs with masses; (d-f) RoIs without masses

false positives, classifying the RoIs between true masses and normal tissue (see Figure 2 for examples of RoIs).

Initially, the RoI image - which contains the suspicious mass - is divided into several local regions. See Figure 3 for an example of a mass image divided into $5 \times$ 5 rectangular regions. Notice that one could use different divisions of different size and shape. From these regions, texture descriptors are independently extracted using LBP and then concatenated to form a global description of the RoI based on the textural information of each region and its spatial distribution. Observe that we could also analyze and combine responses of multiple LBP operators realized with different parameters $(P, R)$. For example, the computation of a set of different LBP operators for some specific regions with higher probability to contain mass information may improve the quality of the final RoI descriptor. This is illustrated in Figure 3 where the internal $3 \times 3$ regions (showed in a dark gray level) are used to compute different LBP operators. Note that a higher weight into the final descriptor is given for these regions. This point will be further discussed in the experimental section.

Following our methodology, the basic LBP histogram is extended into a spatially enhanced histogram which encodes both the local region appearance and the spatial relations (global geometry) of the mass. The RoI image is divided into $m$ small regions $R_{0}, R_{1}, \ldots, R_{m}$ and the spatially enhanced histogram is defined as

$$
H_{i, j}=\sum_{x, y} I\left(f_{l}(x, y)=i\right), \quad(x, y) \in R_{j}
$$

where $i=0, \ldots, n-1, j=0, \ldots, m-1$. In this histogram, the RoI is described on three different levels of locality: the labels for the histogram contain the pixel-level texture patterns, the labels are summed over a small region to produce information on a regional level and finally the regional histograms are concatenated to build a global description of the mass.

The final step of our proposal is the mass classification. For this purpose we use the well-known Support Vector Machines (SVM) technique 14 which performs an implicit mapping of data into a higher dimensional feature space, where linear algebra and geometry can be used to separate data. For our specific problem, SVM with a polynomial kernel is used to provide a membership between RoIs depicting a true mass and RoIs depicting normal parenchyma. In the experimental section we will compare the results obtained by using SVM with those obtained using a Nearest Neighbor (NN) classifier. 


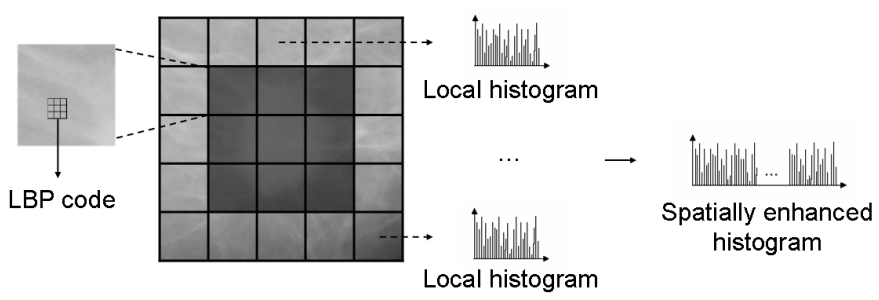

Fig. 3. Strategy for computing the LBP descriptor of an image

\section{Experimental Results}

Our approach has been evaluated using a database of 1792 RoIs extracted from the DDSM mammographic database [15. From this set, 256 depicted a true mass, while the rest 1536 were normal, but suspicious tissue. According to the size of the lesion, we use six different groups of RoI images, corresponding to the following mass sizes intervals: $<10 \mathrm{~mm}^{2},(10-60) \mathrm{mm}^{2},(60-120) \mathrm{mm}^{2},(120-$ 190) $\mathrm{mm}^{2},(190-270) \mathrm{mm}^{2},>270 \mathrm{~mm}^{2}$. The number of masses in each interval were $28,32,37,57,69$, and 33 , respectively. Note we are dealing with different lesion sizes, an important aspect for correctly classifying the masses.

The evaluation of our experiments is done by using a leave-one-out strategy and Receiver Operating Characteristics (ROC) analysis. In the leave-one-out methodology, a specific input RoI is selected and classified according to the model obtained by training the system with the remaining RoIs in the database. This procedure is repeated until all the RoIs have been used as an input image. The SVM classifier provides a numerical value related to the membership of each class. Thus, varying the threshold of this membership it is possible to generate a ROC curve 16. In such analysis, widely used in the medical field, the graphical curve represents the true positive rate as a function of the false positives rate. The percentage value under the curve (known as $A z$ ) is an indication for the overall performance of the observer, and is typically used to analyze the performance of the algorithms.

In order to perform a more global evaluation we compute the $A z$ value for different ratios of number of RoIs depicting masses and number of RoIs depicting normal tissue (from ratio $1 / 1$ to ratio $1 / 6$ ). The idea of analyzing these different ratios is twofold: firstly, to evaluate the performance of our method on different levels of difficulty, and secondly, to compare our proposal with existing methods. Notice that previous works only provide results for specific ratios. Hence, analyzing all these ratios will enable the comparison with them.

As explained in Section 3, some parameters may be optimized in order to obtain the final LBP descriptor. For instance, the number of regions in which a RoI image is divided or the parameters $(P, R)$ used to obtain the LBP responses. With the aim of choosing the number of divisions, we tested the performance of LBP on three different configurations $(3 \times 3,5 \times 5$ and $7 \times 7$ regions $)$ for all the RoI image sizes, all ratios, and using the basic $L B P_{8,1}^{u 2}$ operator. The best result was 


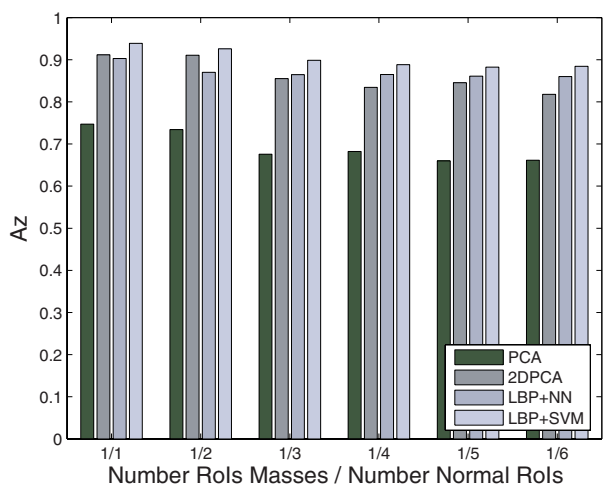

Fig. 4. $A z$ values obtained with the methods PCA, $2 \mathrm{DPCA}, \mathrm{LBP}+\mathrm{NN}$ and LBP+SVM. Each $A z$ value is the mean computed from the results of different RoI image sizes.

obtained when dividing each RoI image into $5 \times 5$ square regions and computing LBP operators for each one. Note that each region size depended on the original RoI image size. Using this configuration we obtained an overall mean $A z$ value of $0.882 \pm 0.051$. With the aim of improving these results and obtaining a more accurate description of the central area, we added a new set of LBP operators for the $3 \times 3$ central regions. In particular, we computed two new LBP responses varying the radius $R$ according to each RoI image size (i.e. $R=1,4$ and 6 was used for the first RoI image size). The global descriptor was then obtained concatenating the 43 histograms of all the regions and LBP operators. For this new configuration, an overall mean $A z$ value of $0.906 \pm 0.043$ was obtained. Note that better results were obtained when including LBP operators with different radius $R$ in the central regions. We then repeated the same experiment varying also the quantization of the angular space $P$ in the basic LBP computation, using $L B P_{8,1}^{u 2}, L B P_{8,2}^{u 2}$, and $L B P_{16,2}^{u 2}$. Similar results were obtained, although, $L B P_{8,1}^{u 2}$ provided the best overall mean results for different RoI image sizes and ratios. This was the final descriptor we used for our experiments since it provided a good trade-off between performance and feature vector length.

Figure 4 shows the obtained mean $A z$ values for each specific ratio when testing our proposal at different RoI image sizes. We include a quantitative comparison with the recent works of Oliver et al. 910, using our database of RoIs. While the first approach is based on using standard PCA, the second introduced a variation on their previous work by using the 2DPCA method [17] instead of the standard PCA technique (we shall refer to these methods as PCA and 2DPCA, respectively). Observe that the performance of our proposal was clearly better than the PCA method. The results were also better than the 2DPCA approach, specially for the cases in which we had smaller ratios $1 / 4$, $1 / 5$ and $1 / 6$. Note also that the use of $2 \mathrm{DPCA}$ itself allowed to obtain better results than the original PCA approach. Using PCA we obtained an overall mean $A z$ value of $0.686 \pm 0.095$ for different RoI image sizes and ratios, using 2DPCA 
Table 1. Az comparison of different methods dealing with mass false positive reduction. We detail the number of RoIs and ratio used in the original works. We also include the results obtained with our LBP+SVM approach.

\begin{tabular}{|c|c|c|c|c|c||c|c|}
\cline { 2 - 8 } \multicolumn{1}{l|}{} & Sahiner [4] & Qian [6] & Chang [7] & Tourassi [8] & Oliver [9] & \multicolumn{2}{|c|}{ LBP+SVM } \\
\hline RoIs & 672 & 800 & 600 & 1465 & 588 & 512 & 1024 \\
\hline Ratio & $1 / 3$ & $1 / 3$ & $1 / 1$ & $\cong 1 / 1$ & $1 / 2$ & $1 / 1$ & $1 / 3$ \\
\hline$A z$ & 0.90 & 0.86 & 0.83 & 0.87 & 0.83 & 0.94 & 0.90 \\
\hline
\end{tabular}

the $A z$ value was $0.868 \pm 0.087$, while using our LBP approach with SVM the $A z$ value was $0.906 \pm 0.043$. Note that we also show the results of LBP when using a Nearest Neighbor (NN) classifier, obtaining an overall mean $A z$ value of $0.871 \pm 0.036$. Observe that LBP with NN provided better performance than 2DPCA for smaller ratios, although the best results were provided by LBP with SVM. Regarding to the behavior when varying the RoI image sizes, we observed that LBP with SVM provided better and more constant results than PCA and 2DPCA methods (see the standard deviations).

We also include in Table 1 a qualitative comparison - in terms of $A z$ value with the rest of the approaches described in Section 1 Note that our efforts were concentrated on obtaining the same ratio of masses used in their experiments. However, we want to clarify that not all the methods used the same databases and therefore our aim is only to provide a general view of the performance of our LBP approach with respect to different strategies. For instance, the works of Chang et al. [7] and Tourassi et al. [8, which used a ratio 1/1, obtained $A z$ values of 0.83 and 0.87 respectively. Note that for this ratio better performances were clearly obtained using our proposal. Similar behavior was observed for the works which used the ratios $1 / 2$ and $1 / 3$. Observe that the difference between the performance reported in the original PCA work of Oliver et al. ( $A z$ of 0.83 ) and the ones showed in our quantitative comparison $(A z$ value of 0.69$)$ is due to the use of different RoI image databases and their particular level of difficulty (the database used in [9] had less images and only 4 different RoI image sizes).

\section{Conclusions}

In this paper we have presented a novel method for mass false positive reduction based on textural features. First of all, a global descriptor for each RoI is obtained by analyzing its spatial textural information. Our approach divides a RoI image into small regions and computes local texture descriptions using local binary patterns. The combination of these local descriptors in a spatially enhanced histogram provides our final feature descriptor. Afterwards, these descriptors are used to classify the RoIs between true masses and normal parenchyma. Our experiments have shown firstly that LBP features are effective and efficient for false positive reduction at different RoI image sizes, and secondly than even when using different ratios of number of RoIs with masses and number of RoIs with normal tissue it is possible to obtain reliable results. 


\section{Acknowledgments}

This work was partially supported by grants TIN2006-08035 and IdIBGi-UdG 91060080.

\section{References}

1. Bray, F., McCarron, P., Parkin, D.M.: The changing global patterns of female breast cancer incidence and mortality. Breast Cancer Research 6, 229-239 (2004)

2. Rangayyan, R.M., Ayres, F.J., Desautels, J.E.L.: A review of computer-aided diagnosis of breast cancer: Toward the detection of subtle signs. Journal of the Franklin Institute 344(3-4), 312-348 (2007)

3. Nishikawa, R.M., Kallergi, M.: Computer-aided detection, in its present form, is not an effective aid for screening mammography. Med. Phys 33, 811-814 (2006)

4. Sahiner, B., Chan, H.P., Wei, D., Petrick, N., Helvie, M.A., Adler, D.D., Goodsit, M.M.: Image feature selection by a genetic algorithm: Application to classification of mass and normal breast tissue. Med. Phys 23, 1671-1684 (1996)

5. Christoyianni, I., Koutras, A., Dermatas, E., Kokkinakis, G.: Computer aided of breast cancer in digitized mammograms. Comp. Med. Imag. and Graph. 26, 309319 (2002)

6. Qian, W., Sun, X., Song, D., Clarke, R.A.: Digital mammography - wavelet transform and kalman-filtering neural network in mass segmentation and detection. Acad. Radiol. 8(11), 1074-1082 (2001)

7. Chang, Y.H., Hardesty, L.A., Hakim, C.M., Chang, T.S., Zheng, B., Good, W.F., Gur, D.: Knowledge-based computer-aided detection of masses on digitized mammograms: A preliminary assessment. Med. Phys 28(4), 455-461 (2001)

8. Tourassi, G.D., Vargas-Vorecek, R., Catarious, D.M., Floyd, C.E.: Computerassisted detection of mammographic masses: A template matching scheme based on mutual information. Med. Phys 30(8), 2123-2130 (2003)

9. Oliver, A., Martí, J., Martí, R., Bosch, A., Freixenet, J.: A new approach to the classification of mammographic masses and normal breast tissue. IAPR Int. Conf. on Patt. Rec. 4, 707-710 (2006)

10. Oliver, A., Lladó, X., Martí, J., Martí, R., Freixenet, J.: False positive reduction in breast mass detection using two-dimensional PCA. In: Lect. Not. in Comp. Sc., vol. 4478, pp. 154-161 (2007)

11. Ahonen, T., Hadid, A., Pietikainen, M.: Face detection with local binary patterns: Application to face recognition. IEEE Trans. Pattern Anal. Machine Intell. 28(12), 2037-2041 (2006)

12. Ojala, T., Pietikainen, M., Maenpaa, T.: Multiresolution gray-scale and rotation invariant texture classification with local binary patterns. IEEE Trans. Pattern Anal. Machine Intell. 24(7), 971-987 (2002)

13. Ojala, T., Pietikainen, M., Harwood, D.: A comparative-study of texture measures with classification based on feature distributions. Patt. Rec. 29(1), 51-59 (1996)

14. Vapnik, V.: Statistical Learning Theory. John Wiley \& Sons, New York (1998)

15. Heath, M., Bowyer, K., Kopans, D., Moore, R., Kegelmeyer, P.J.: The Digital Database for Screening Mammography. In: Int. Work. on Dig. Mammography, pp. 212-218 (2000)

16. Metz, C.E.: Evaluation of digital mammography by ROC analysis. In: Int. Work. on Dig. Mammography, pp. 61-68 (1996)

17. Yang, J., Zhang, D., Frangi, A.F., Yang, J.: Two-dimensional PCA: a new approach to appearance-based face representation and recognition. IEEE Trans. Pattern Anal. Machine Intell. 26(1), 131-137 (2004) 